ICASE Report No. 97-25



\title{
TOWARDS PERFECTLY ABSORBING BOUNDARY CONDITIONS FOR EULER EQUATIONS
}

\section{Ehtesham Hayder \\ Fang Q. Hu \\ M. Yousuff Hussaini}

NASA Contract No. NAS1-19480

May 1997

Institute for Computer Applications in Science and Engineering NASA Langley Research Center

Hampton, VA 23681-0001

Operated by Universities Space Research Association

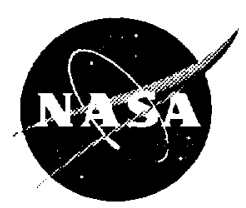

National Aeronautics and Space Administration

Langley Research Center Hampton, Virginia 23681-0001 



\title{
Towards Perfectly Absorbing Boundary Conditions for Euler Equations
}

\author{
M. Ehtesham Hayder ${ }^{1}$ \\ Institute for Computer Applications in Science and Engineering \\ Mail Stop 403, NASA Langley Research Center \\ Hampton, VA 23681-0001 \\ Fang Q. Hu \\ Department of Mathematics and Statistics \\ Old Dominion University, Norfolk, VA 23529 \\ M. Yousuff Hussaini \\ Program in Computational Science and Engineering \\ Florida State University, Tallahassee, FL 32:306-3075
}

\begin{abstract}
In this paper, we examine the effectiveness of absorbing layers as non-reflecting computational boundaries for the Euler equations. The absorbing-layer equations are simply obtained by splitting the governing equations in the coordinate directions and introducing absorption coefficients in each split equation. This methodology is similar to that used by Berenger for the numerical solutions of Maxwell's equations. Specifically, we apply this methodology to three physical problems - shock-vortex interactions, a plane free shear flow and an axisymmetric jet - with emphasis on acoustic wave propagation. Our numerical results indicate that the use of absorbing layers effectively minimizes numerical reflection in all three problems considered.
\end{abstract}

\footnotetext{
${ }^{1}$ This research was supported by the National Aeronautics and Space Administration under NASA Contract No. NAS1-19480 while the author was in residence at the Institute for Computer Applications in Science and Engineering (ICASE), NASA Langley Research Center, Hampton, VA 23681-0001
} 


\section{Introduction}

The proper treatment of computational boundaries is crucial for any numerical solution to a set of partial differential equations which governs fluid motion or wave propagation in a medium. Various techniques have been developed to minimize the reflection of out-going waves. A review can be found in Givoli (1991). Numerical boundary conditions based on the characteristics of the relevant linearized equations and their asymptotic solutions in the far field have been widely used. However, such boundary conditions are not satisfactory if the outflow is nonlinear or involves multi-directional waves. As a possible remedy, a buffer zone abutting the computational boundary, in which the governing equations are modified, and whose role is to absorb the incident waves, has been proposed. In this buffer zone. the modifications have the effect of either removing or damping reflected waves oriented back towards the computational domain. Naturally, the buffer zone solutions themselves need not necessarily be physical, and they serve only to prevent contamination of the solution in the physical domain of interest by the reflections from the computational boundaries. Various types of buffer zone techniques have been used in flow simulations. For example, Colonius et al.(1993) used buffer zones in which the solutions were filtered. In a different approach. Ta'asan and Nark (1995) modified the governing equations in the buffer zone to change the orientation of the characteristics, and make the flow supersonic at the exit plane. Recently, Berenger(1994) proposed a very effective Perfectly Matched Layer technique for Maxwell's equations. In this approach, the equations governing the so-called matched layer are split into subcomponents with damping terms which absorb the incident waves almost perfectly. Following Berenger, $\mathrm{Hu}$ (1996), developed an analogous technique for the linearized Euler equations, and provided analytical results for the case of uniform flow.

In this paper, we follow the operator splitting principle of Berenger (1994) and $\mathrm{Hu}$ (1996) for the equations governing what we call the absorbing layers and examine their effectiveness in the case of shock-vorticity wave interactions, a plane free-shear layer and an axisymmetric jet. The emphasis is on the effectiveness of the the computational boundaries in handling wave propagation including sound waves. It is shown that the absorbing layer technique is very effective for all three physical problems. The next section describes briefly the numerical models used in this study, followed by the section on results and conclusion.

\section{Numerical Models}

\subsection{Shock Wave Interactions}

To verify the applicability of the absorbing boundary condition technique to shock-turbulence and shock-vortex interaction problems, we choose the numerical model of Erlebacher, Hussaini and Shu (1997). This model solves the fully nonlinear compressible Euler equations along with a time evolution equation for the shock motion for the purpose of fitting the shock. The outflow boundary conditions which minimizes wave reflection back into the domain of computation are of crucial importance for such problems as they involve long-time 
integrations. The present case focuses on the interaction of a single vorticity wave with a shock wave, and the results of course carry over simply to a randomly distributed wave system. The two dimensional Euler equations are written as

$$
\begin{gathered}
\frac{\partial \rho}{\partial t}+u \frac{\partial \rho}{\partial x}+v \frac{\partial \rho}{\partial y}=-\rho\left(\frac{\partial u}{\partial x}+\frac{\partial v}{\partial y}\right) \\
\frac{\partial u}{\partial t}+u \frac{\partial u}{\partial x}+v \frac{\partial u}{\partial y}=-\frac{1}{\rho} \frac{\partial p}{\partial x} \\
\frac{\partial v}{\partial t}+u \frac{\partial v}{\partial x}+v \frac{\partial v}{\partial y}=-\frac{\partial p}{\partial y} \\
\frac{\partial p}{\partial t}+u \frac{\partial p}{\partial x}+v \frac{\partial p}{\partial y}=-\gamma p\left(\frac{\partial u}{\partial x}+\frac{\partial v}{\partial y}\right)
\end{gathered}
$$

The computational domain has the shock as a boundary on the left and an outflow boundary on the right, and is periodic in the other direction. Fourth order Runge-Kutta scheme is used for time integration, and the spatial derivatives are discretized by a compact $6^{\text {th }}$ order scheme.

In the absorbing layer at the right boundary, the Euler equations are split into a locally one-dimensional set with artificial damping terms. Consider the pressure equation, for example, in computational space:

$$
\frac{\partial p}{\partial t}=-a_{1} \frac{\partial p}{\partial X}-a_{2} \frac{\partial p}{\partial Y}-\gamma p\left(\frac{\partial w_{1}}{\partial X}+\frac{\partial w_{2}}{\partial Y}\right)
$$

After operator splitting and addition of damping terms, the pressure equation becomes

$$
\begin{aligned}
& \frac{\partial p_{1}}{\partial t}=-a_{1} \frac{\partial p}{\partial X}-\gamma p \frac{\partial w_{1}}{\partial X}-\sigma_{X} p_{1} \\
& \frac{\partial p_{2}}{\partial t}=-a_{2} \frac{\partial p}{\partial Y}-\gamma p \frac{\partial w_{2}}{\partial Y}-\sigma_{Y} p_{2}
\end{aligned}
$$

in the absorbing layer. Here, $w_{1}$ and $w_{2}$ are velocity components in $x$ - and $y$-directions, $a_{1}$ and $a_{2}$ are contravariant velocity components (which include the effect of grid motion) in computational space, and $p=p_{1}+p_{2}$. Locally one-dimensional equations for the other variables are constructed in a similar manner. The damping factor $\sigma_{X}$ is zero in a layer parallel to the $X$ direction; similarly $\sigma_{Y}$ is zero in a layer parallel to the $Y$ direction (see Figure 1). However, in the corner region both these damping factors are positive. 


\subsection{Free Shear Layer}

In order to evaluate the performance of the absorbing-layer technique in the case of inviscid instability waves, we solve the linearized Euler equations in a Cartesian $(x, y)$ coordinate system. We study the evolution of a Kelvin-Helmholtz instability wave as it propagates downstream and impinges on the absorbing layers. In this case, the $x$-momentum equation reads

$$
\frac{\partial u}{\partial t}+\bar{U} \frac{\partial u}{\partial x}+\frac{d \bar{U}}{d y} v+\frac{1}{\bar{\rho}} \frac{\partial p}{\partial x}=0
$$

where

$\bar{U}=\frac{1}{2}\left[\left(U_{1}+U_{2}\right)+\left(U_{1}-U_{2}\right) \tanh (y)\right]$. Absorbing layers are used at the upper, lower and right boundaries. Again, the afore-mentioned operator splitting in the absorbing layer leads to two $x$-momentum equations:

$$
\begin{gathered}
\frac{\partial u_{1}}{\partial t}+\sigma_{x} u_{1}+\bar{l} \frac{\partial u}{\partial x}+\frac{1}{\bar{\rho}} \frac{\partial p}{\partial x}=0 \\
\frac{\partial u_{2}}{\partial t}+\sigma_{y} u_{2}+\frac{d \bar{U}}{d y} v=0
\end{gathered}
$$

where $u=u_{1}+u_{2}$. All other equations are treated similarly. These equations are solved by a low-dissipation and low-dispersion Runge-Kutta scheme which is formally fourth order accurate (Hu, Hussaini and Manthey, 1996).

For the nonlinear case one uses again an approximate time independent mean flow to split the Euler equations in the absorbing layer. Thus the stream-wise velocity for two dimensional flows is decomposed into three components:

$$
u=\bar{U}+u_{1}+u_{2}
$$

where $\bar{U}$ is the mean velocity as in the linear case. Then the $x$-momentum equation is written as

$$
\frac{\partial u}{\partial t}+u u_{x}+v u_{y}+\frac{1}{\rho} p_{x}=0 .
$$

This equation is then split into two equations as

$$
\begin{gathered}
\frac{\partial u_{1}}{\partial t}+\sigma_{x} u_{1}=-\frac{1}{\rho} p_{x}-u u_{x}+\frac{1}{\bar{\rho}} P_{x}+\bar{U} \bar{U}_{x} \\
\frac{\partial u_{2}}{\partial t}+\sigma_{y} u_{2}=-v u_{y}
\end{gathered}
$$

All other equations in the absorbing layer are similarly derived. 


\subsection{Axisymmetric Jet}

The compressible axisymmetric Euler equations for the jet in the weak conservation form are : $Q_{t}+F_{z}+G_{r}=S$, where, in the linearized case,

$$
\begin{gathered}
Q=r\left(\begin{array}{c}
\rho \\
m_{z} \\
m_{r} \\
E
\end{array}\right), \quad F=r\left(\begin{array}{c}
m_{z} \\
p+2 m_{z} U_{z}-\rho U_{z}^{2} \\
m_{z} U_{y}+m_{r} U_{z}-\rho U_{z} U_{r} \\
(p+E) U_{z}+\left(m_{z}-\rho U_{z}\right) H
\end{array}\right) \\
S=\left(\begin{array}{c}
0 \\
0 \\
p \\
0
\end{array}\right), \quad G=r\left(\begin{array}{c}
m_{r} \\
m_{z} U_{r}+m_{r} U_{z}-\rho U_{z} U_{r} \\
p+2 m_{r} U_{r}-\rho U_{r}^{2} \\
(p+E) U_{r}+\left(m_{r}-\rho U_{r}\right) H
\end{array}\right) \\
p=(\gamma-1)\left[E-\left(m_{z} U_{r}+m_{r} U_{z}\right)+\frac{\rho}{2}\left(U_{z}^{2}+U_{r}^{2}\right)\right]
\end{gathered}
$$

In the above equations, $p, \rho, m_{z}, m_{r}, E$ denote the fluctuating components of pressure, density, axial and radial momentum, total energy and $H$ is the mean enthalpy. These equations have been linearized around the mean velocity $\left(U_{r}, U_{z}\right)$ represented by an error function that fits experimental measurements. The interior equations are simply split into

$$
Q_{t}+F_{z}=0, \quad Q_{t}+G_{r}=S
$$

and they are modified in the absorbing layer as

$$
Q_{t}^{1}+F_{z}=-\sigma_{z} Q^{1}+S^{1}, \quad Q_{t}^{2}+G_{r}=-\sigma_{r} Q^{2}+S^{2}
$$

where $Q=Q^{1}+Q^{2}$ and $S=S^{1}+S^{2}$. (We used $S^{1}=0$ in this study).

In the nonlinear case, the vectors $Q, S, F$, and $(i$ are defined as follows.

$$
\begin{gathered}
Q=r\left(\begin{array}{c}
\rho \\
\rho u \\
\rho v \\
E
\end{array}\right), \quad S=\left(\begin{array}{c}
0 \\
0 \\
p \\
0
\end{array}\right), \\
F=r\left(\begin{array}{c}
\rho u \\
\rho u^{2}+p \\
\rho u v \\
\rho u H
\end{array}\right), \quad G=r\left(\begin{array}{c}
\rho v \\
\rho u v \\
\rho v^{2}+p \\
\rho v H
\end{array}\right) .
\end{gathered}
$$


We use the fourth-order MacCormack method which has been successfully used in earlier studies by Hayder et al. (1996) to solve the linearized Euler equations, and by Hayder et al. (1993) and Mankbadi et al. (1994) to solve the Navier Stokes equations. The equations are linearized before splitting to obtain the equations for the absorbing layer. Thus, we get

$$
\begin{gathered}
\left(Q^{1}-Q_{0}^{1}\right)_{t}+\left(F-F_{0}\right)_{z}=-\sigma_{z}\left(Q^{1}-Q_{0}^{1}\right) \\
\left(Q^{2}-Q_{0}^{2}\right)_{t}+\left(G-\left(G_{0}\right)_{r}=-\sigma_{r}\left(Q^{2}-Q_{0}^{2}\right)+\left(S-S_{0}\right)\right.
\end{gathered}
$$

where the subscript 0 denotes mean quantities.

\section{Results}

In the case of the shock-vorticity wave interaction, we consider specifically the following simple wave

$$
\begin{gathered}
u-U_{1}=\epsilon U_{1} 2 \sqrt{2} \frac{k_{y}}{k} \cos \left(k_{x} x+k_{y} y-U_{1} t\right) \\
v=-\epsilon U_{1} 2 \sqrt{2} \frac{k_{y}}{k} \cos \left(k_{x} x+k_{y} y-U_{1} t\right) \\
\rho=p=T=1
\end{gathered}
$$

as the upstream condition ahead of the shock. $U_{1}$ is the upstream mean velocity normal to the undisturbed shock, $k_{y}=k \sin \theta, k_{x}=k \cos \theta$ (where $k$ is upstream wavenumber), and $\epsilon=0.001$ measures the intensity of the wave. Our standard interior domain is 7.4 units long with 185 uniformly spaced grid points. We used 16 points on the coordinate axis parallel to the shock. An absorbing layer abuts the right outflow boundary. We introduce damping gradually in order to minimize any reflections due to the discretization in the absorbing layer. Unless otherwise mentioned, we use $\theta=30^{\circ}, \mathrm{K}=2$, and 25 grid points (= 1 unit in length) in the buffer layer for our computations. A snapshot of pressure in the interior domain at $t=20$ is presented in Figure 2, which shows how well the out-going waves are absorbed with little reflection. To measure the contamination due to reflection, the solutions are compared with a reference solution obtained by computing the flow in a much larger domain with the same spatial and temporal resolution. We follow this methodology for all problems in this study. Figure 3 compares axial variation in pressure for two different size buffers against the large domain solution at $t=20$. Because of modifications to the governing equations, the solution in the buffer layer is irrelevant. The solution in the interior domain for a buffer with 25 points is visually indistinguishable from the larger domain solution. In Figure 4, we show the rms error $(E)$ in pressure at the ordinate four grid points upstream of the interface between the computational domain and the absorbing layer as a function of the layer thickness measured in the number of equidistant points. The error $E$ is defined as

$$
E=\frac{100}{\left|p_{\max }^{r}\right|} \sqrt{\frac{\sum_{j=1}^{N}\left(p^{r}-p\right)^{2}}{N}}
$$


where $p^{r}$ is the pressure from the reference solution, $\left|p_{\text {max }}^{r}\right|$ is its maximum amplitude and $N$ is the number of grid points in the $y$-direction ( $N=16$ in the current context). $E$ measures the numerical error in the solution, which includes both direct and induced errors due to the interaction of residual reflections from the outflow boundary with the flow and the shock. As expected, $E$ decreases as the layer width is increased. In Figures 5 and 6 , we show the dependence of numerical errors on the angle of incidence $(\theta)$ and the wave number $(\mathrm{k})$. The buffer layer is more effective at lower incidence angle and wavenumbers, although we notice some cross-overs in our numerical experiments. At later times, a fraction of the reflections from the outflow boundary propagates upstream. These waves can then reflect back and forth, and cause what we call induced errors. These sometimes constitute a significant portion of the errors shown in Figures 4-6 at later times.

The results for the free-shear layer are obtained for upper and lower stream mean velocities, normalized by the speed of sound, equal to $U_{1}=0.6$ and $U_{2}=0.2$ respectively. The eigenfunctions of the Kelvin-Helmholtz instability wave given by the linear stability theory are forced at the inflow, with a maximum amplitude $\epsilon$ equal to 0.01 . We solve the linearized Euler equations and the solution agrees with the linear theory very well in eigenfunction and growth rate comparisons. In Figure 7 , snapshots of axial velocity (Fig 7 a) and pressure (Fig Th) are shown, and in Figure 8 we present the amount of reflection as a function of the layer thickness. We observe that for 10 points in the absorbing layer, the amount of reflection (measured four grid points away from the buffer layer boundary) is less than .03\% of the amplitude of the reference pressure fluctuation from the large domain solution. We also solve nonlinear Euler equations where the nonlinearity in the flow is significant. The inflow excitation amplitude $(\epsilon)$ is kept at 0.01 , but the interior domain is three times longer. All other flow parameters are the same as in the linear case. The error in pressure four grid points away from the buffer layer in shown in Figure 9. We needed a larger buffer layer for the nonlinear flow simulations. At time equal to 3000 , errors with 30 and 50 points in the buffer layer were $3.5 \%$ and $4 \%$ respectively. Intuitively one expects that a buffer layer to be more effective if nonlinear effects are smaller. This may be the principal reason for larger errors in Figure 9 compared to Figure 8. The effect of nonlinearity is also shown in Figure 10 , where we compare errors for simulations with two different levels of excitation $\epsilon$ with a buffer of 50 grid points.

Finally, for the case of the excited axisymmetric jet, we assume the mean Mach number to be 0.6. At the inflow, we extrapolated one characteristic variable corresponding to the outgoing acoustic wave from the interior and computed the other three characteristic variables at time $t$ using $[\rho, u, v, p]=\epsilon \operatorname{Re}\left(\hat{q} \epsilon^{i \omega t}\right)$, where $\hat{q}=[\hat{\rho}, \hat{u}, \hat{v}, \hat{p}]$ is the eigenfunction given by the linear stability theory, $t=10^{-4}, \omega=1.05$. A snapshot of pressure is shown in Figure 11. The rms pressure error $(E)$ in the immediate neighborhood (four points away from the buffer layer) of the layer interface is plotted in Figure 12 for time equal to upto 50 . This error becomes quasi-periodic and the maximum error for 25 grid points in the absorbing layer is about $0.015 \%$. Our results for the nonlinear Euler equations are shown in Figure 13. The domain size is 10 units long for both linearized and nonlinear Euler simulation of the 
excited jet. The physical parameters are the same for both the linearized and the nonlinear Euler equations for the jet calculations.

\section{Conclusions}

In conclusion, we find the performance of the absorbing-layer technique in the cases of three physical problems (using three different numerical algorithms) is quite satisfactory. This technique offers a viable alternative to the traditional boundary treatments based on the linearized characteristics or asymptotic solutions in the far field, and also other types of buffer layers. It also promises to be accurate and inexpensive for aeroacoustic computations. Further studies are warranted to put this methodology on a firm footing.

\section{References}

Berenger, J-P, "A Perfectly Matched Layer for the Absorption of Electro-magnetic Waves", Journal of Computational Physics, 114: 185-200, 1994.

Colonius, T., Lele, S. K. and Moin, P., "Boundary Conditions for Direct Computation of Aerodynamic Sound Generation", AIAA Journal, 31: 1574-1582, 1993.

Erlebacher, G., Hussaini, M. Y. and Shu, C., "Interaction of a Shock with a Longitudinal Vortex", ICASE report 96-31, to appear in .Journal of Fluid Mechanics, 1997.

Givoli, D., "Non-reflecting Boundary Conditions", Journal of Computational Physics, 94: $1-29,1991$.

Hayder, M. E. Turkel, E. and Mankbadi, R. R., "Numerical Simulations of a High Mach Number Jet Flow", AIAA paper 93-0653, NASA TM 105985, 1993.

Hayder, M. E., Zhou, Y. and Rubinstein, R., "Numerical Simulation of the Mixing Noise in Turbulent Flows", Proceedings of the Fluid Engineering Division Summer Meting, FED-Vol. 238, ASME, pp 479-484, 1996.

Hu, F. Q., "On absorbing boundary conditions for linearized Euler equations by a Perfectly Matched Layer", Journal of Computational Physics, 129: 201-219, 1996.

Hu, F. Q., Hussaini, M. Y. and Manthey, J. L., "Low-Dissipation and Low-Dispersion Runge- 
Kutta Schemes for Computational Acoustics" Journal of Computational Physics, 124: 177$191,1996$.

Mankbadi. R. R., Hayder, M. E. and Povinelli, L. A., "The Structure of Supersonic Jet Flow and Its Radiated Sound" AIAA Journal, 32: 897-906, 1994.

Ta'asan, S. and Nark, D. M., "An Absorbing Buffer Zone Technique for Acoustic Wave Propagation", AIAA paper 95-0164, 1995. 


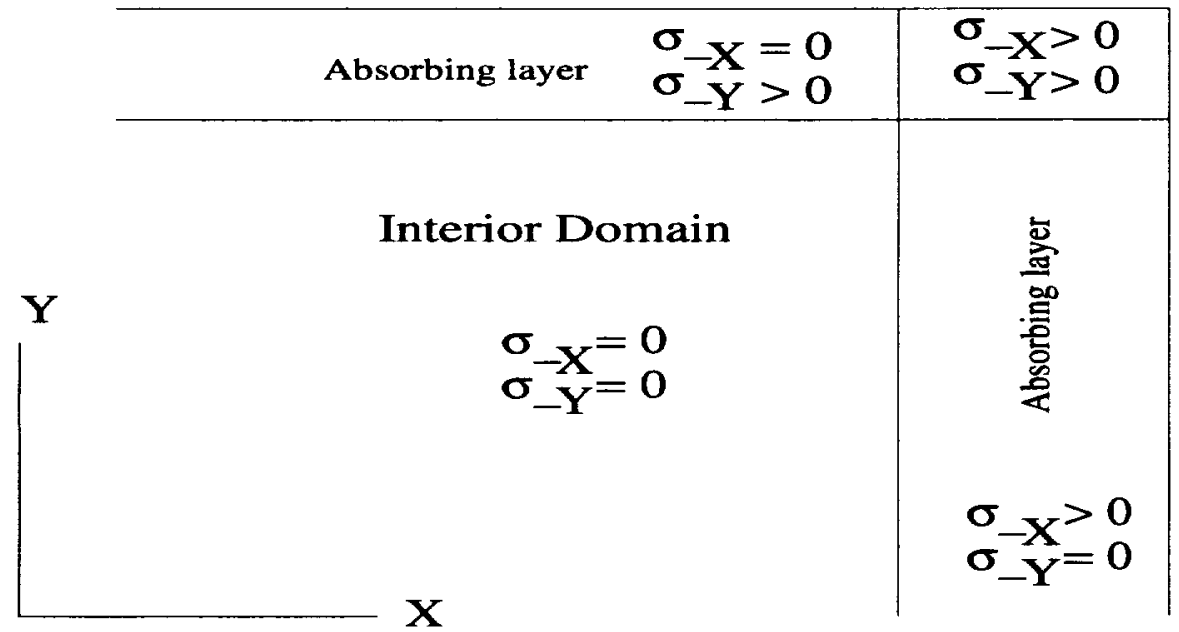

Figure 1: Schematics of absorbing layers

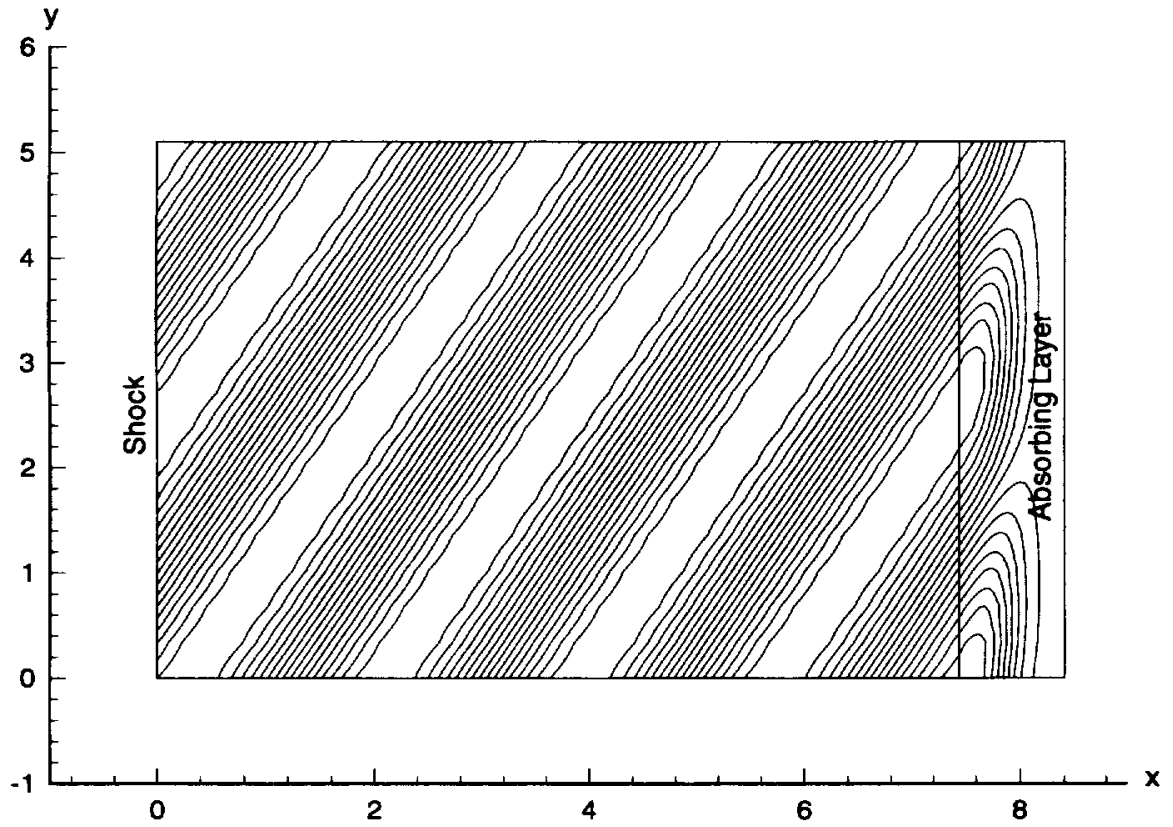

Figure 2: A snapshot of pressure 




Figure 3: Effect of buffer layer

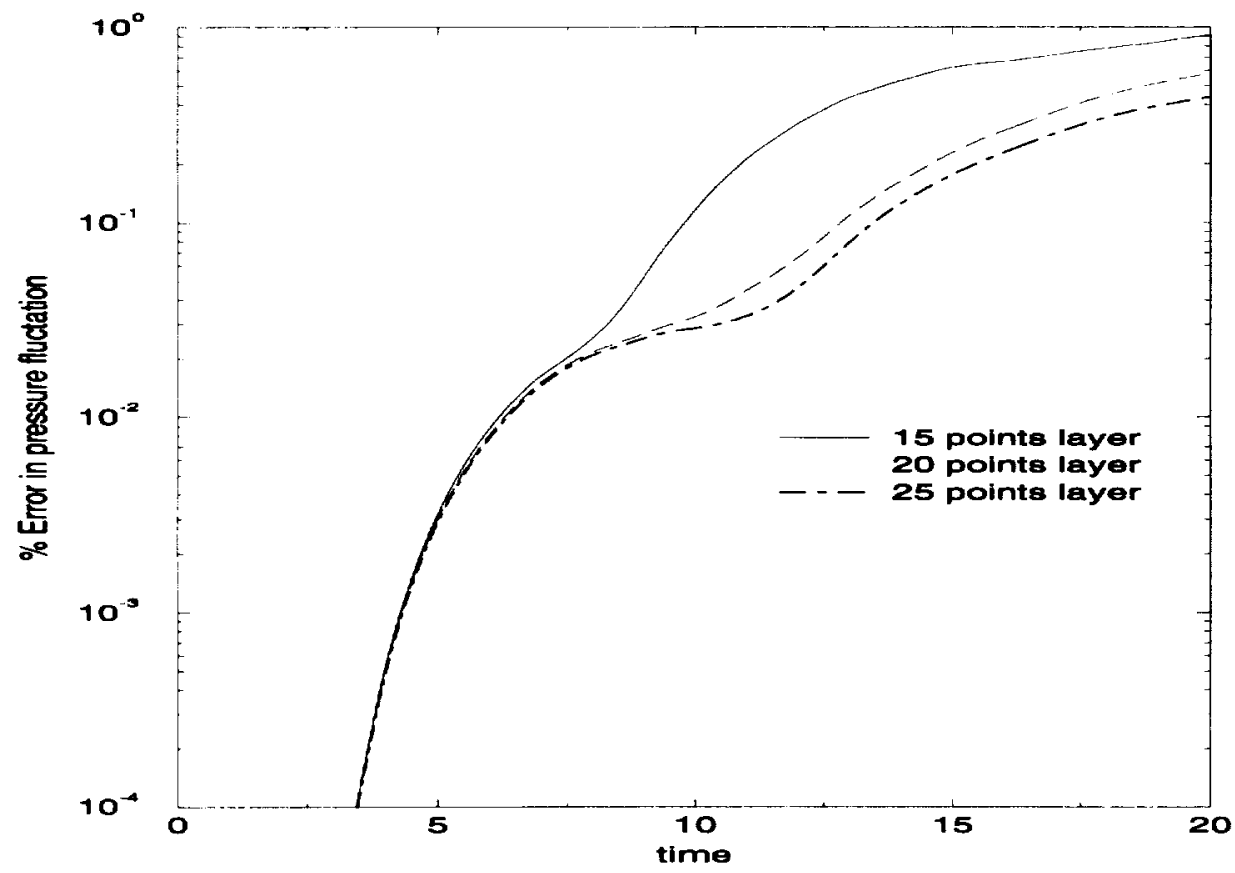

Figure 4: Effect of layer thickness 




Figure 5: Angle dependence

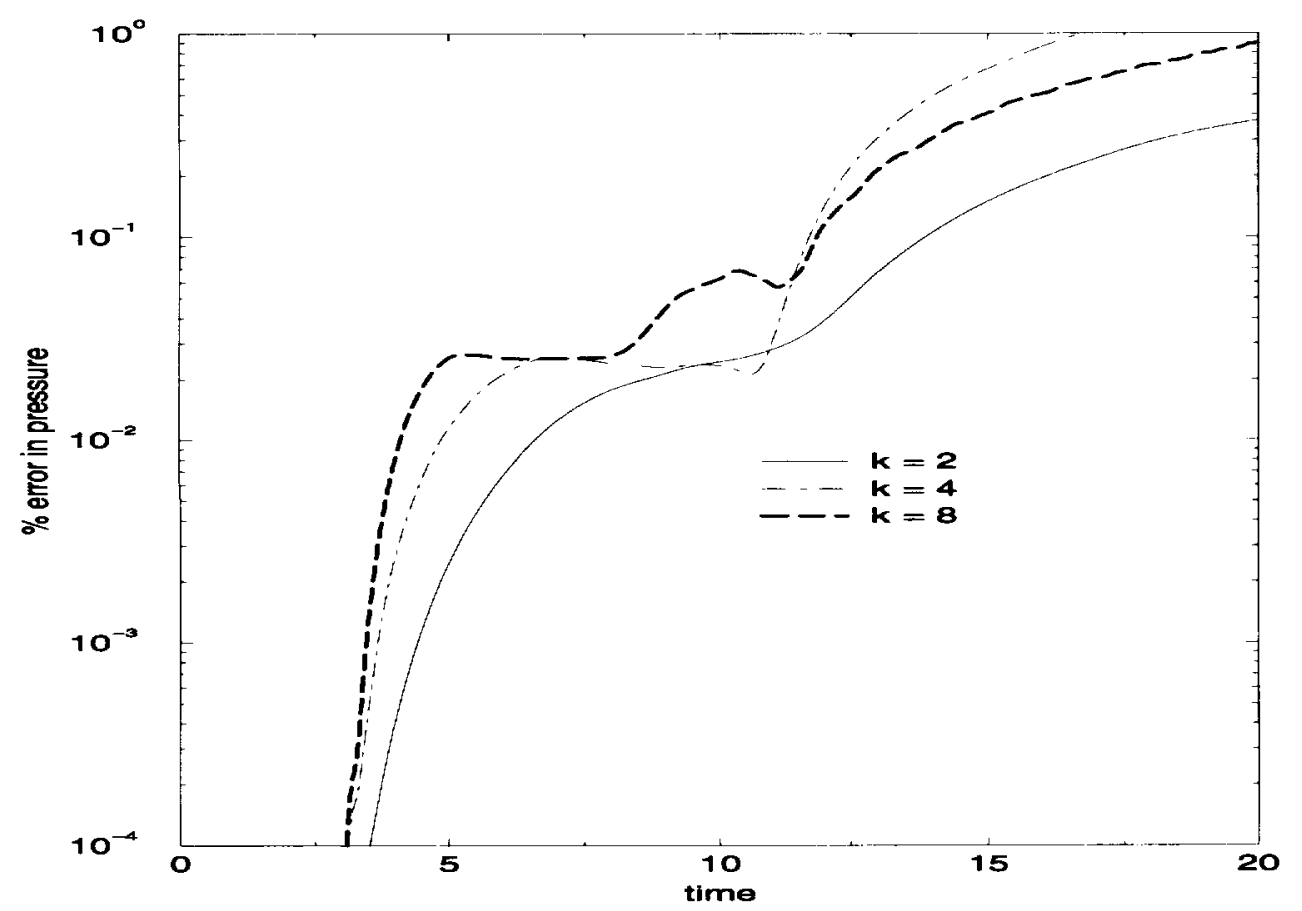

Figure 6: Wave number dependence 

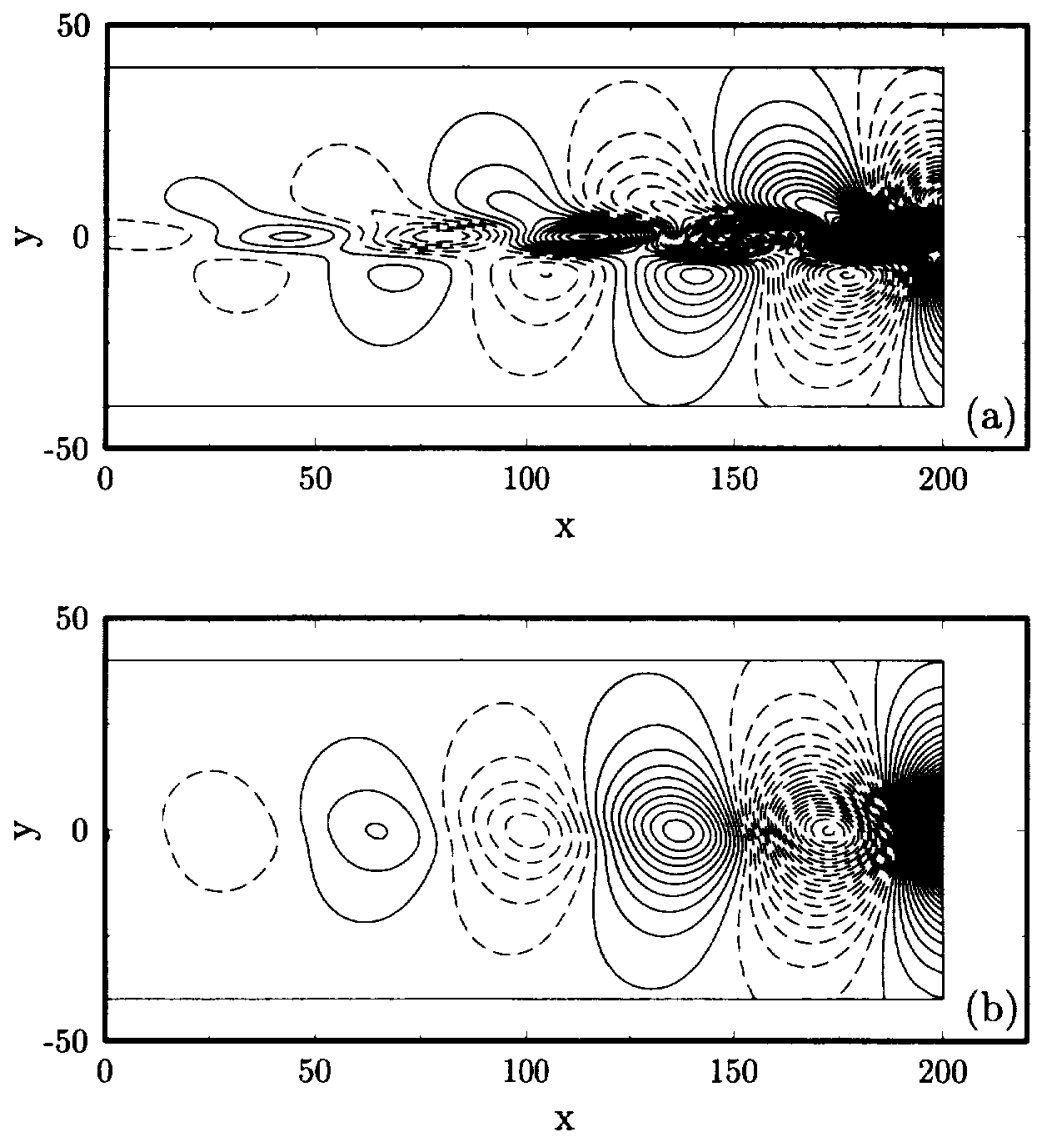

Figure 7 : Velocity and pressure contours in a free shear layer

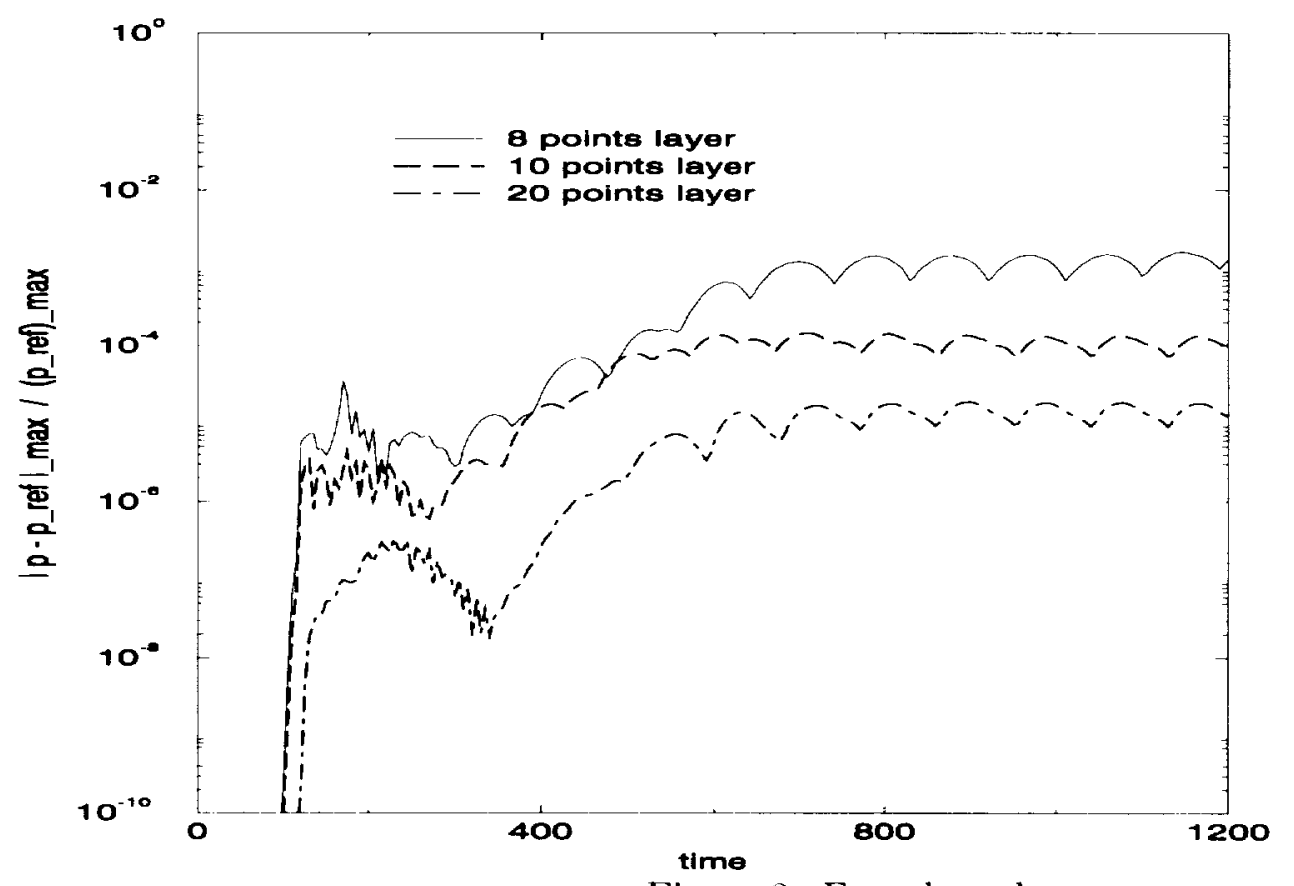

Figure 8: Free shear layer 


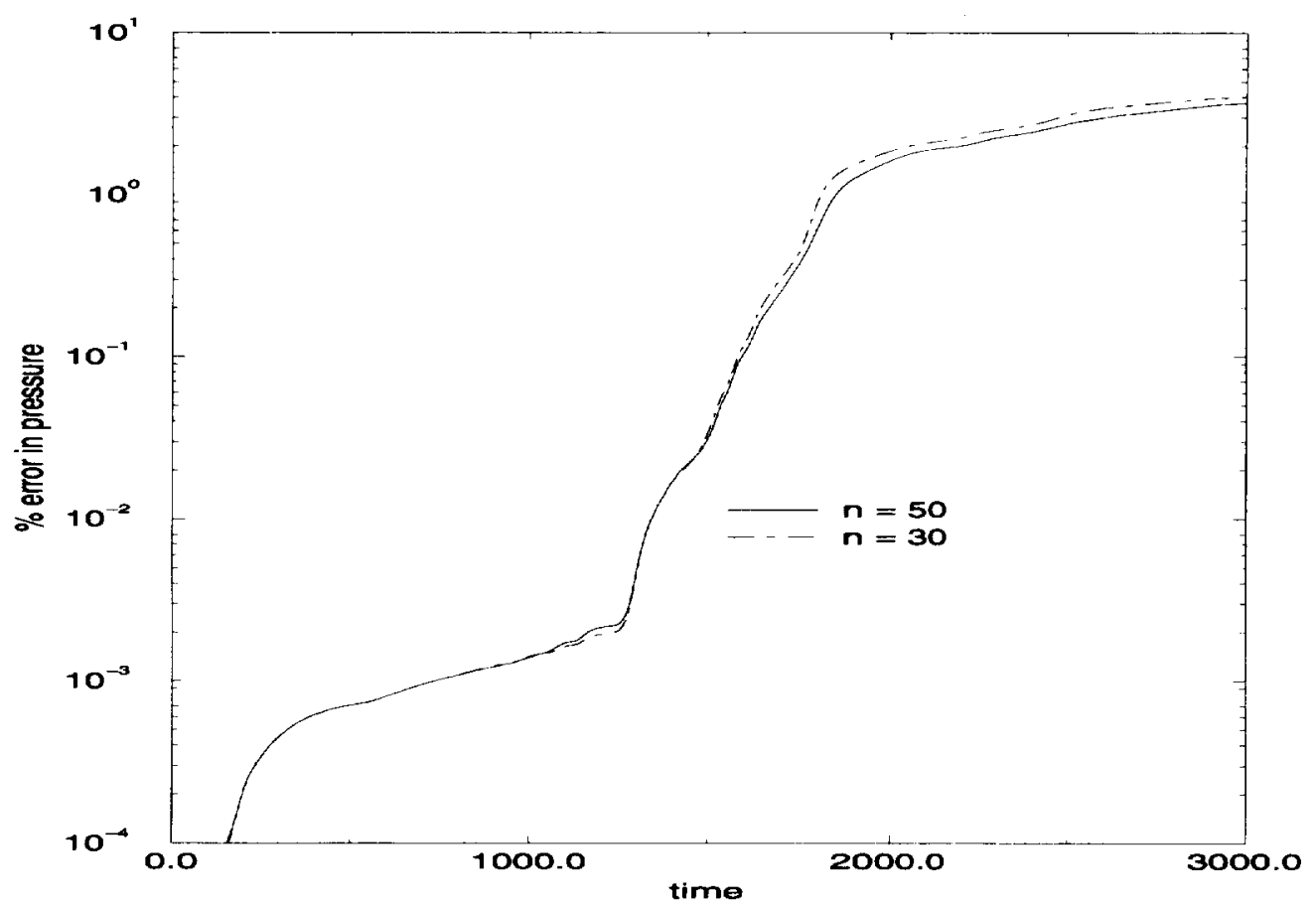

Figure 9: Nonlinear free shear layer

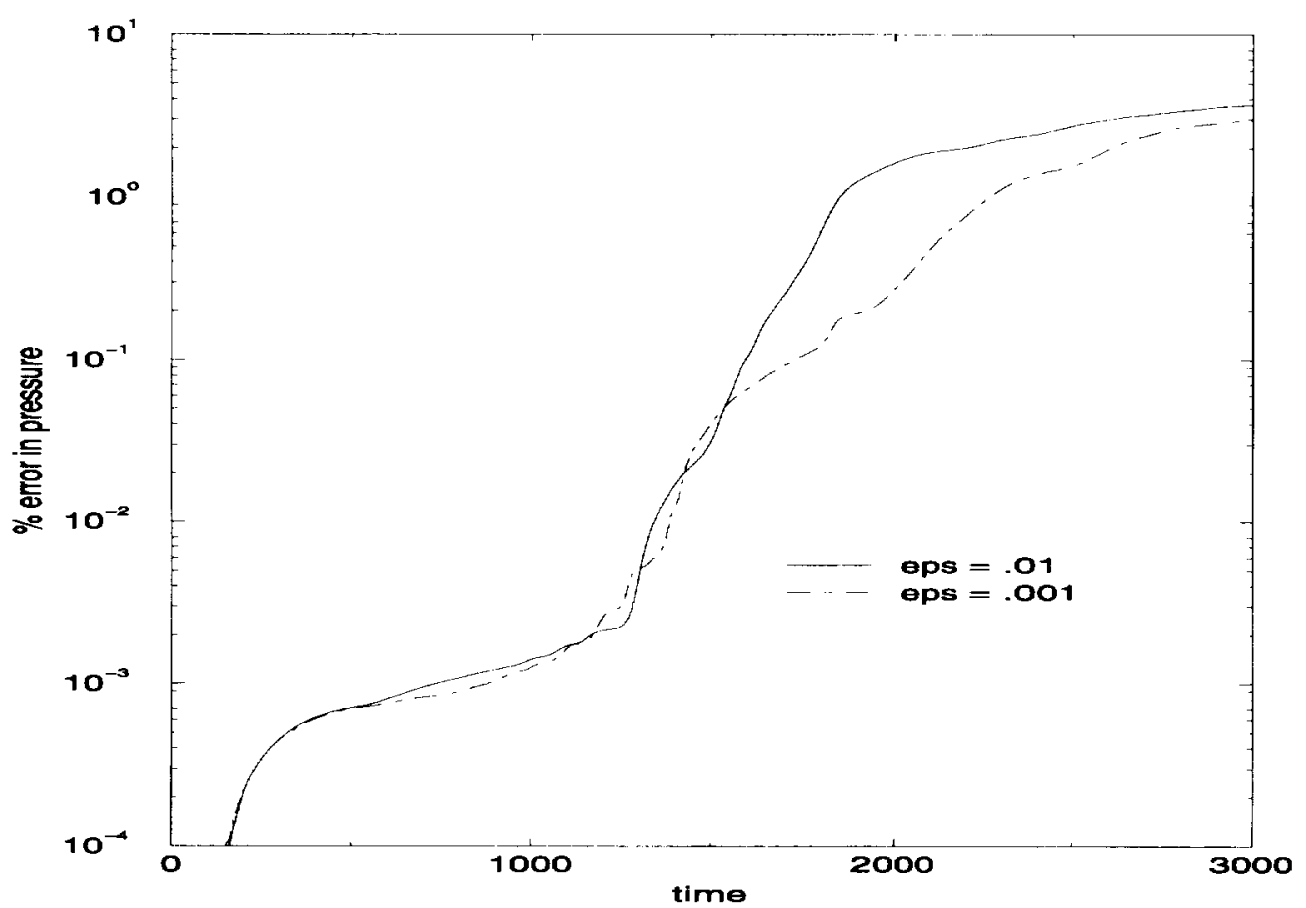

Figure 10: Effect of excitation level 


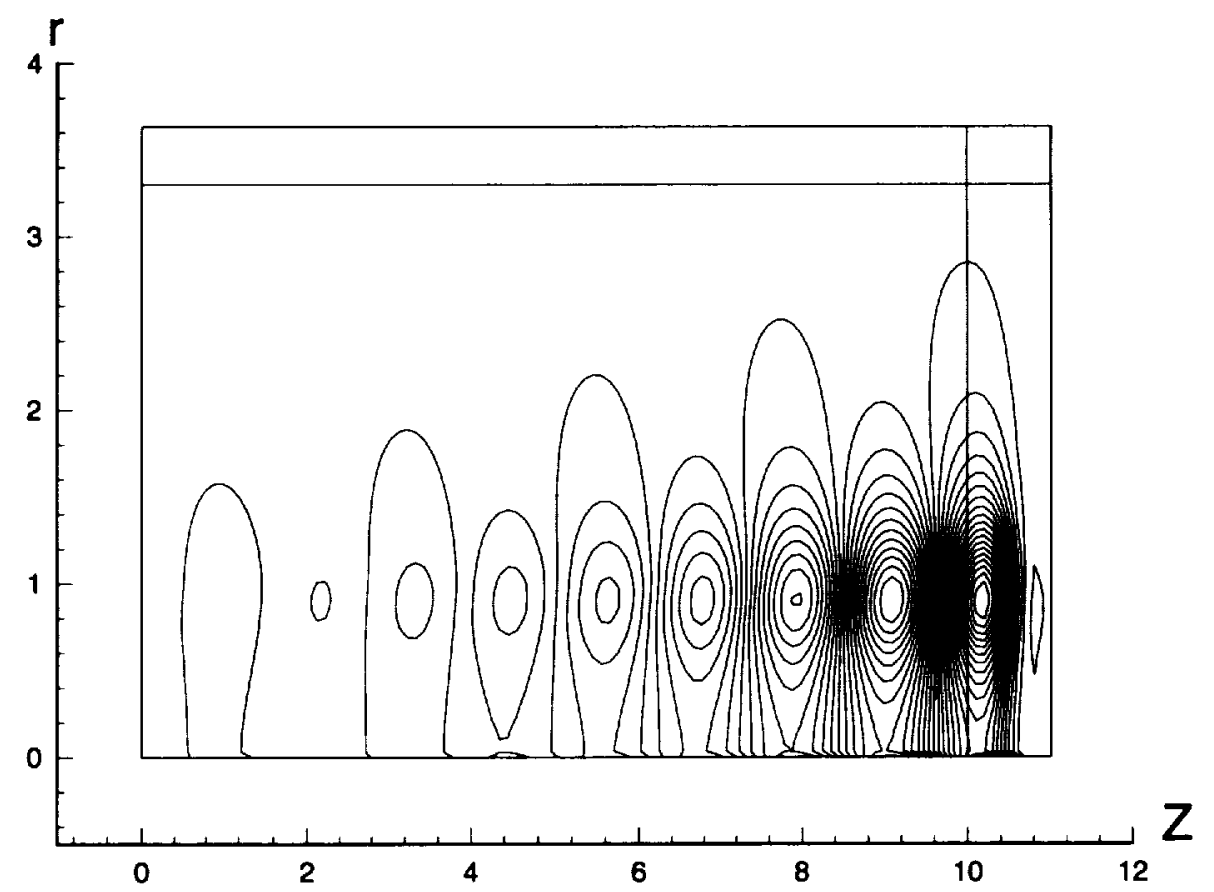

Figure 11: A snapshot of pressure in the jet

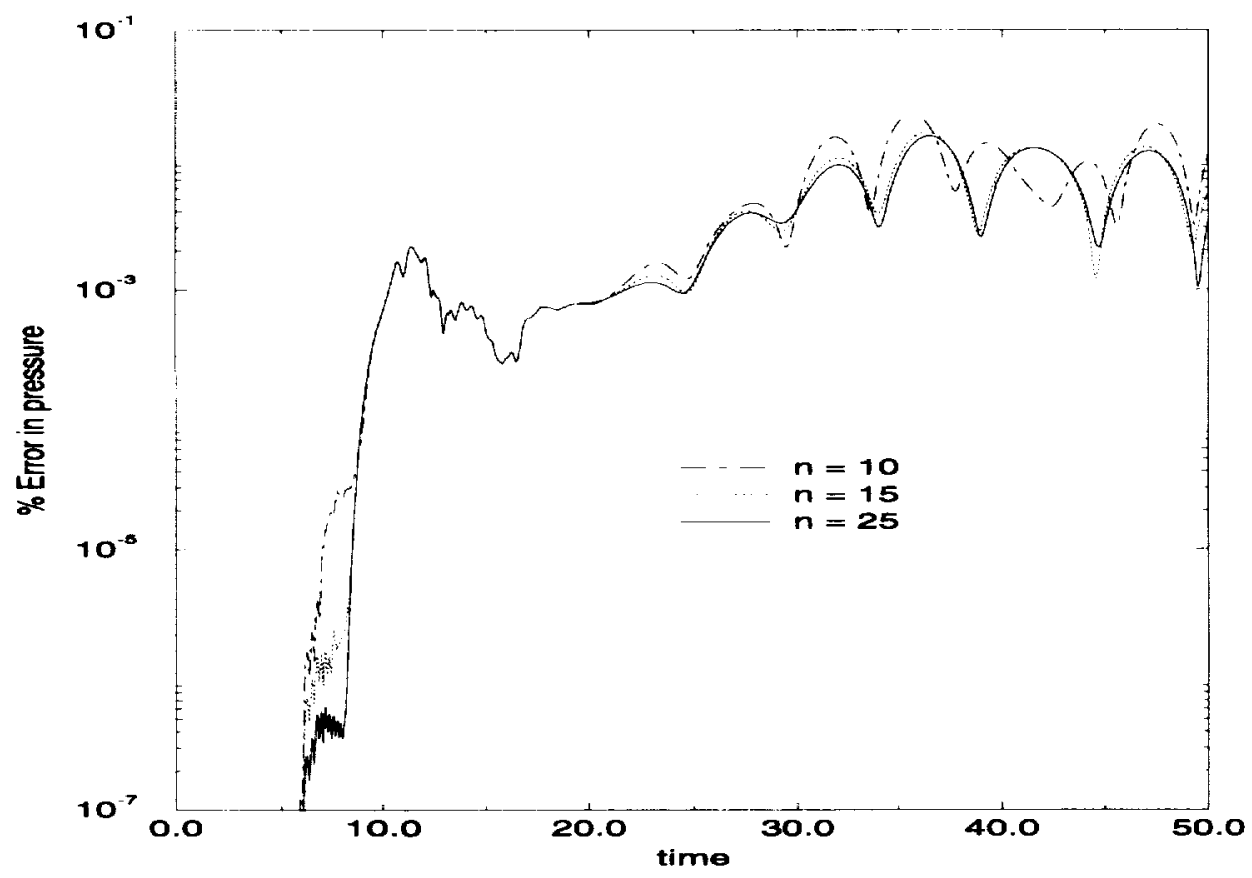

Figure 12: Axisymmetric jet (Linearized Euler) 


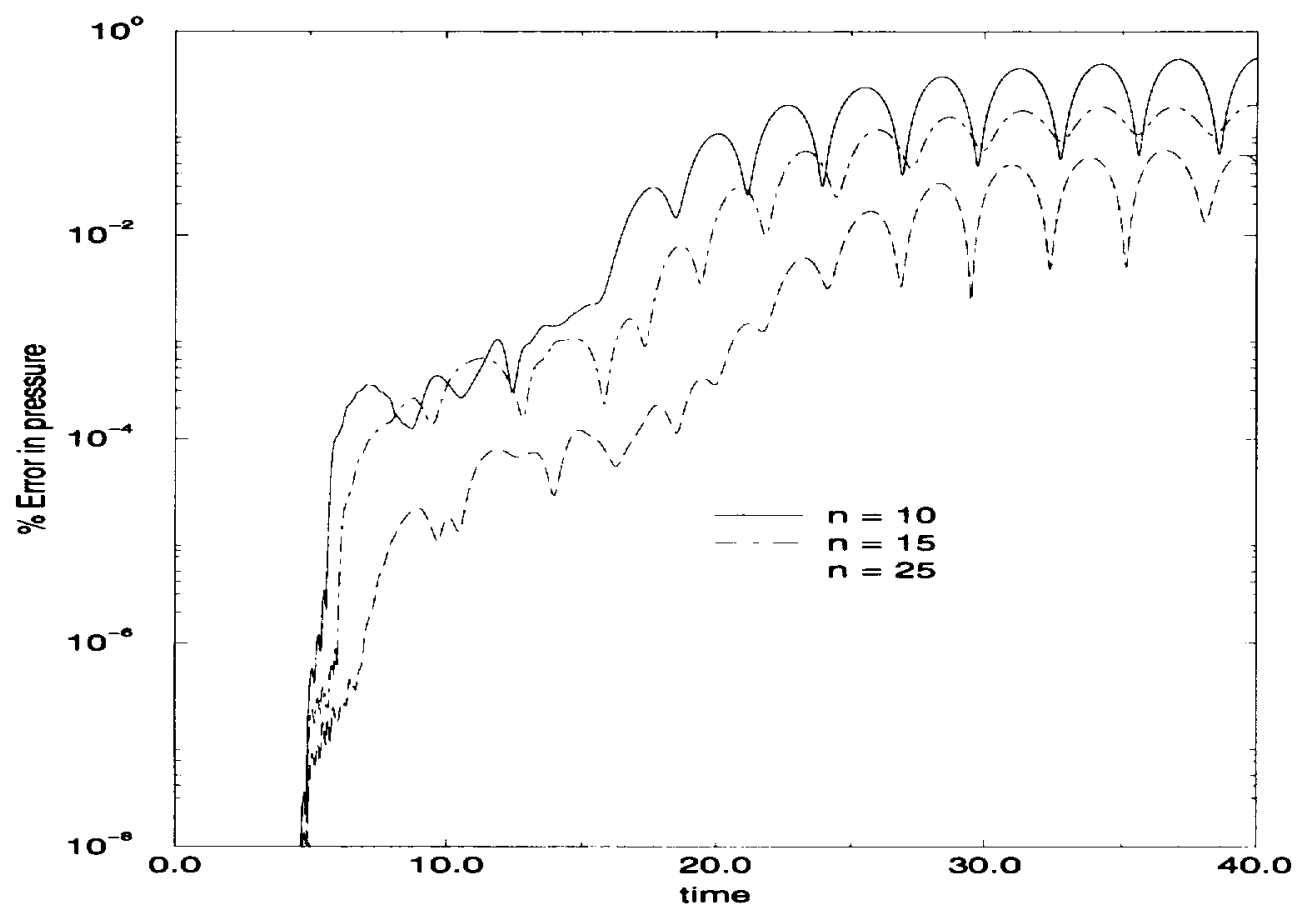

Figure 13: Axisymmetric jet (Euler) 




\begin{tabular}{|c|c|c|}
\hline \multicolumn{2}{|c|}{ REPORT DOCUMENTATION PAGE } & $\begin{array}{l}\text { Form Approved } \\
\text { OMB No. 0704-0188 }\end{array}$ \\
\hline \multicolumn{3}{|c|}{  } \\
\hline 1. AGENCY USE ONLY(Leave biank) & $\begin{array}{l}\text { 2. REPORT DATE } \\
\text { May } 1997\end{array}$ & $\begin{array}{l}\text { D DATES COVERED } \\
\text { rt }\end{array}$ \\
\hline \multicolumn{2}{|c|}{$\begin{array}{l}\text { 4. TITLE AND SUBTITLE } \\
\text { TOWARDS PERFECTLY ABSORBING BOUNDARY } \\
\text { CONDITIONS FOR EULER EQUATIONS }\end{array}$} & $\begin{array}{l}\text { 5. FUNDING NUMBERS } \\
\text { C NAS1-19480 } \\
\text { WU } 505-90-52-01\end{array}$ \\
\hline \multicolumn{3}{|l|}{$\begin{array}{l}\text { 6. AUTHOR(S) } \\
\text { M. Ehtesham Hayder } \\
\text { Fang Q. Hu } \\
\text { M. Yousuff Hussaini }\end{array}$} \\
\hline \multicolumn{2}{|c|}{$\begin{array}{l}\text { 7. PERFORMING ORGANIZATION NAME(S) AND ADDRESS(ES) } \\
\text { Institute for Computer Applications in Science and Engineering } \\
\text { Mail Stop 403, NASA Langley Research Center } \\
\text { Hampton, VA 23681-0001 }\end{array}$} & $\begin{array}{l}\text { 8. PERFORMING ORGANIZATION } \\
\text { REPORT NUMBER } \\
\text { ICASE Report No. } 97-25\end{array}$ \\
\hline \multicolumn{2}{|c|}{$\begin{array}{l}\text { 9. SPONSORING/MONITORING AGENCY NAME(S) AND ADDRESS(ES) } \\
\text { National Aeronautics and Space Administration } \\
\text { Langley Research Center } \\
\text { Hampton, VA } 23681-0001\end{array}$} & $\begin{array}{l}\text { 10. SPONSORING/MONITORING } \\
\text { AGENCY REPORT NUMBER } \\
\text { NASA CR-201689 } \\
\text { ICASE REPOrt No. } 97-25\end{array}$ \\
\hline \multirow{2}{*}{\multicolumn{3}{|c|}{$\begin{array}{l}\text { 11. SUPPLEMENTARY NOTES } \\
\text { Langley Technical Monitor: Dennis M. Bushnell } \\
\text { Final Report } \\
\text { Submitted to AIAA Journal (13th AIAA CFD Conference) }\end{array}$}} \\
\hline & & \\
\hline \multirow{2}{*}{\multicolumn{2}{|c|}{$\begin{array}{l}\text { 12a. DISTRIBUTION/AVAILABILITY STATEMENT } \\
\text { Unclassified-Unlimited } \\
\text { Subject Category } 34,64\end{array}$}} & 12b. DISTRIBUTION CODE \\
\hline & & \\
\hline \multicolumn{3}{|c|}{$\begin{array}{l}\text { 13. ABSTRACT (Maximum } 200 \text { words) } \\
\text { In this paper, we examine the effectiveness of absorbing layers as non-reflecting computational boundaries for the } \\
\text { Euler equations. The absorbing-layer equations are simply obtained by splitting the governing equations in the } \\
\text { coordinate directions and introducing absorption coefficients in each split equation. This methodology is similar to } \\
\text { that used by Berenger for the numerical solutions of Maxwell's equations. Specifically, we apply this methodology to } \\
\text { three physical problems - shock-vortex interactions, a plane free shear flow and an axisymmetric jet - with emphasis } \\
\text { on acoustic wave propagation. Our numerical results indicate that the use of absorbing layers effectively minimizes } \\
\text { numerical reflection in all three problems considered. }\end{array}$} \\
\hline
\end{tabular}

